International Journal of Engineering \& Technology, $7(2.20)(2018) 230-235$
International Journal of Engineering \& Technology
SPC
Website: www.sciencepubco.com/index.php/IJET
Research paper

\title{
Investigation on Interference Mitigation Schemes for Next Generation Cellular Communications
}

\author{
V. Thrimurthulu ${ }^{*}$, N.S. Murti Sarma ${ }^{2}$ \\ ${ }^{1}$ Research Scholar, Electronics and Communication Engineering, Rayalaseema University, Kurnool, A.P., India. \\ ${ }^{2}$ Professor, Dept. of ECE, Sreenidhi Institute of Science \& Technology, Ghatkesar, Hyderabad, T.S, India. \\ *Corresponding author E-mail:vtmurthy.v@gamil.com
}

\begin{abstract}
The fanciful quick development and exponential increment of advanced mobile phones the remote info activity request is additional within the returning years. That forces reexamining current remote cell arranges thanks to the shortage of the acc essible vary. 2 noteworthy difficulties for advancing future Evolution (LTE) systems area unit to accomplish improved cell scope and framework limit contrasted and broadband Code Division Multiple Access (WCDMA) framework. Powerful use of radio assets and thick vary use is at the middle to realize these objectives. Be that because it might, thick repeat use might increment between cell electric resistance, that so extraordinarily restricts the limit of shoppers within the framework. Between cell obstruction will limit general framework execution as so much as output and preternatural productivity, significantly for the shoppers set at the cell edge region. later on, cautious administration of between cell obstructions finishes up vital to boost LTE framework execution. during this paper, obstruction alleviation plans for LTE downlink systems area unit investigated. The eNB and also the MobileFemto as each provide similar assets and transfer speed. This has created AN electric resistance issue from the downlink sign s of every alternative to their UEs. this examination has adjusted a adept repeat use conspire that worked powerfully finished separation and accomplished increased outcomes within the flag quality and output of Macro and Mobile-Femto UE once contrasted with past electric resistance administration plans e.g. Partial Frequency use factor1 (NoFFR-3) and uncomplete Frequency use factor3 (FFR-3).
\end{abstract}

Keywords: LTE, WCDMA, UE, eNB, Femtocell and FFR.

\section{Introduction to interference}

Impedance in cell frameworks may be a standout amongst the foremost well-known problems that influence the framework execution. Albeit each transmission profit is meted out a district of return, directors shall expand the output by sharing the vary between BSs. The LTE framework is planned therefore every eNB utilizes the complete BW with a return employ of one. causation Femtocells to reinforce the vary productivity; in any case, these new conferred elements within the LTE organize style might build Associate in Nursing obstruction issue. on these lines, the key check is to reinforce the vary use, whereas relieving the cochannel obstruction of Macro-UEs and Femto-UEs. it's to be aforementioned that, there area unit various co-channel electrical resistance things that farthest purpose the task and causation of Femtocells. The developing interest of giving ubiquitous broadband internet access on versatile systems has forced the requirement of making OFDMA (Orthogonal Frequency Division Multiple Access) based mostly remote cell systems, let's say, 4G systems. one in every of the important difficulties for advancing LTE systems is to expand organize limit [1]. In spite of the very fact that thick return employ brings concerning noteworthy framework limit amendment, it likewise astonishingly debases the execution of the framework attributable to the growth in electrical resistance caused by conterminous cells [2]. In cell transportable correspondence framework, for the foremost half 2 kinds of electrical resistance should be thought of, let's say, intra-cell obstruction and between cell obstruction. The intra-cell electrical resistance meddling versatile terminal is in Associate in Nursing indistinguishable cell from appeared in Fig.1. The overflow transmission between contiguous channels within a cell brings concerning intra-cell electrical resistance. In between cell electrical resistance (ICI) the meddling transportable terminal is in conterminous cell as appeared in Fig.2. ICI is caused by the use of an analogous return divert in neighboring cells [3].

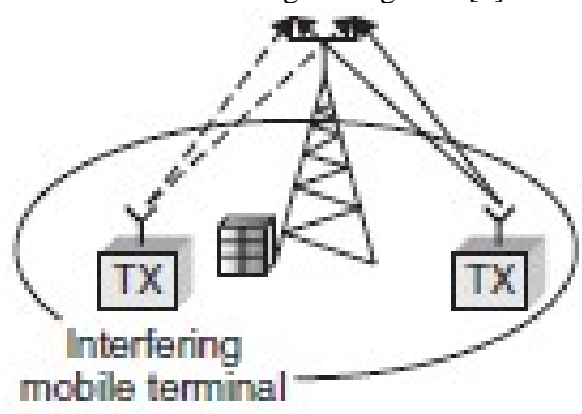

Fig. 1: Intra-cell interference

In LTE downlink (DL), OFDMA radio access innovation is used wherever the subcarriers ar usually orthogonal to every alternative, inferring that there's no intra-cell electrical resistance. Be that because it might, ICI will restrain framework execution relating to outturn and phantom effectiveness, notably for purchasers placed at the cell edge. on these lines, cautious administration of between cell obstruction is essential in LTE to 
boost framework performance. To relieve between cell electrical resistance (ICI), some procedures ar used amid the transmission or once the gathering of the flag. ICI alleviation ways are often named obstruction randomisation, electrical resistance cancelation and obstruction evasion. within the principal procedure, some cell specific scrambling, interleaving or unfold vary systems are often used to decrease electrical resistance. The obstruction is sent haphazardly among all purchasers, let's say, utilizing pseudorandom scrambling once channel cryptography. during this method, the phone edge purchasers will not typically endure solid ICI amid the total UTC frame. In electrical resistance cancelation, the meddling sign is recovered through flag handling and therefore the assessed meddling sign is subtracted from the gotten flag that's needed flag and obstruction flag [5].

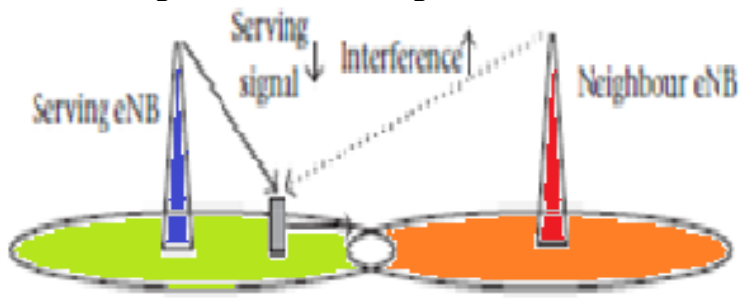

Fig. 2: Inter-cell interference

In electrical resistance goldbricking, the assignment of various assets like time, recurrence, and power is controlled to create flag to obstruction and clamor proportion, SINR; on these lines turnout, for cell edge shoppers and to confirm ICI are within middle of as so much as doable. electrical resistance goldbricking system guarantees higher administrations for shoppers placed at cell edge while not yielding cell focus clients' turnout. The advantages of utilizing every of those ICI moderation procedures specific higher than area unit essentially unrelated. afterward, mix of those plans is predicted in up and coming back frameworks. The relieving electrical resistance a no-hit usage of radio assets, as an example, control, composed parcel booking assumes exceptionally noteworthy half. during this paper, we tend to essentially focus on electrical resistance goldbricking plans that area unit additional known with the decilitre (Downlink) LTE systems. a brief review of obstruction organization is likewise given.

\section{Inter-cell interference (ICI)}

In LTE, the tiniest unit of radio quality which will be meted out to a consumer for data transmission amid parcel designing is termed physical quality piece (PRB). Radio assets area unit characterised in time-recurrence space as appeared in higher piece of Fig.3. A period/recurrence radio quality that ranges over one schedule gap of zero.5ms within the time space and one sub-channel $(180 \mathrm{KHz})$ of twelve subcarriers within the repeat area is thought as quality sq. (RB). The Rb sets (in time area unita) are selected to a UE for data transmission in an exceedingly TTI (Transmission Time Interval $=1 \mathrm{~ms})$. Between cell electric resistance is caused attributable to crashes between RBs that area unit employed by varied cells all the whereas $[6,7]$. The PRBs reusing by UEs placed at near cells lead to ICI in OFDMA frameworks [7]. At the purpose once a consumer moves from serving eNB and seems to be nearer to its neighboring eNB, the got SINR (Signal to Interference and Noise Ratio) debases because the in demand got flag management diminishes and therefore the ICI increments. The result of between cell electric resistance in LTE deciliter is investigated by ascertaining the gotten SINR of UEm on the RBn (Resource Block $\mathrm{n}$ ) as per the condition (1).

$$
\operatorname{SINR}_{m, n}=\frac{p_{n}^{l} H_{m, n}^{l}}{k \neq l p_{n}^{k} H_{m, n}^{k} \delta_{n}^{k}+P_{N}}
$$

Where, SINR $_{\mathrm{m}, \mathrm{n}}$ is the SINR of $\mathrm{UE}_{m}$ on $\mathrm{RB}_{n}$
$P_{n}^{l}$ is the transmission power from the serving cell $l$ on RB

$H_{m, n}^{k}$ is the channel gain from the cell $l$ to $\mathrm{UE}_{m}$ on $\mathrm{RB}_{n}$

$P_{N} \quad$ is the noise power

$\delta^{k}{ }_{n}$ is indicator which is set to 1 or 0 to specify whether the neighboring cell $k$ allocates $\mathrm{RB}_{n}$ to its UEs or not.

\section{Resource block collision $=$} Interference
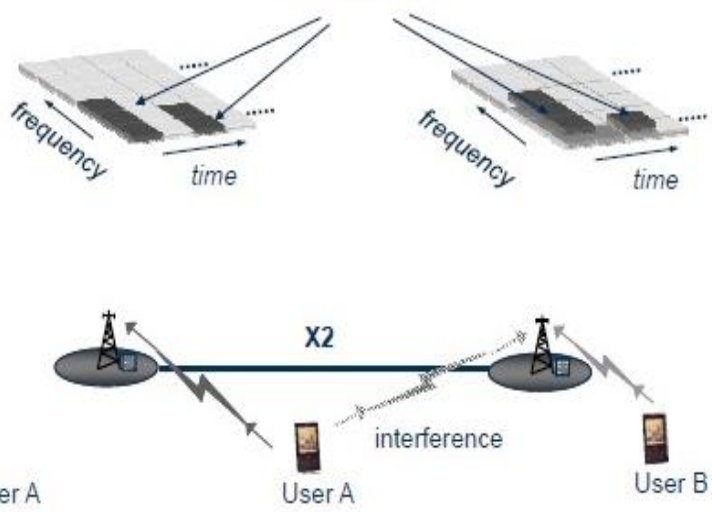

User B

Empty Resource Block

Fig. 3: Fundamentals of inter-cell interference in OFMDA systems

A scope of energy and repetition portion techniques is received for cell focus and cell edge shoppers to alleviate ICI. In spite of the very fact that Associate in Nursing enlargement in transmit power will enhance the flag to resistivity and clamor proportion, it would altogether expand the overall obstruction of the framework. during this manner for each UE, particularly cell edge consumer (CEU), Associate in Nursing enlargement in client's transmit management forces a competition over the overall framework execution. during this manner, completely different power allotment plans ar projected to induce Associate in Nursing exchange off between the accomplished SINR and taking place resistivity thus because the framework's execution is increased [8]. Considering the third circumstance once ICI increments (when the neighbor cells assign an identical $\mathrm{Rb}$ to their clients), completely different $\mathrm{Rb}$ distribution plans ar received as ICI alleviation plots within which the goal of the plans is to reduce ICI and sustain the upper transcendental productivity at the same time. it's in addition seen from the equation(1) that if the serving cell chooses RBn to transmit data, the ICI is lessened noticeably if the contiguous cells do not carried out identical RBn to their shoppers i.e. whenever . The portion coarseness is Associate in Nursing quality sq. or a chunk of accessible transmission capability. The origination of saving explicit bits of the information transmission for CCU and CEU to evade obstruction is known as repetition use system.

\section{Interference avoidance schemes}

In cell organize, distinctive power and repetition distribution plans area unit sent to take care of a strategic distance from the result of ICI therefore because the framework's unearthly proficiency may be created strides. In varied ICI relief plans, repetition recycle strategy is taken because the elementary thought. These repetition recycle arrangement calculations mean to boost the SINR, Associate in Nursingd should satisfy the ability limitation of each cell by making certain that the transmit energy of an eNB is not surpassing the foremost extreme permissible cutoff. in sight of your time scale, ICI evasion, the repetition recycle plans may be classified as static, semi-static and dynamic plans. Static assignment plans will work on a moderately expansive continuance $[10,11]$. In static arrange, the quality distribution for each phone is resolved amid radio arrangement and simply end of the day corrections area unit created amid organize task. Consequently, the ability levels and therefore the arrangement of sub-transporters parceled out for each cell and cell area unitas are 
static (settled). In semi-static approach, a bit of the rubidium assignment is predefined and alternate RBs distributions saved for cell edge shoppers area unit powerfully modified. Timescale of reallocation is in seconds or a number of hundred milliseconds. In distinctive arrange, quality allotment is more and more invigorated in lightweight of the kinds of system conditions. Dynamic assignments area unit done when a short span amount $[2,11]$. within the incidental subsections talked concerning the plans.

\section{Static schemes}

Regardless of clear contrasts between varied repetition recycle plans, all plans ought to indicate the arrangement of subtransporters assigned for each cell/division, the ability level at that every channel works, and therefore the cell locales (i.e. cell focus or cell edge) during which the arrangement of sub-bearers (channels) area unit used. completely different repetition recycle plans verify distinctive qualities for these parameters [6].

\section{Conventional frequency recycle}

The easiest repetition recycle conspire is to use a repetition recycle issue one (RF1). In RF1, the combination accessible transmission capability is reused in each cell while not representing any limitations on management portion or repetition quality utilization that as appeared in Fig.4 (a). With this arrange, high framework limit i.e. high pinnacle data rate may be accomplished. even so, between cell obstruction, notably at cell edges, is expanded that so considerably constrains the execution of cell-edge shoppers. during this manner, the final ghastly productivity degrades. In recycle issue 3 (RF3), the combination transfer speed is isolated into 3 equivalent and orthogonal subgroups and therefore the sub-groups area unit assigned to cells such close cells faithfully convey numerous frequencies that as appeared in Fig.4(b). This arrange prompts bring down between cell resistivity. Be that because it could, as each cell is utilizing thirty third of combination accessible transfer speed, there's a considerable limit misfortune.
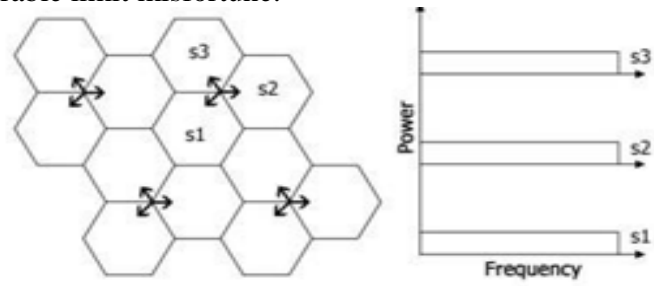

Fig. 4(a): Conventional frequency planning with reuse factor 1
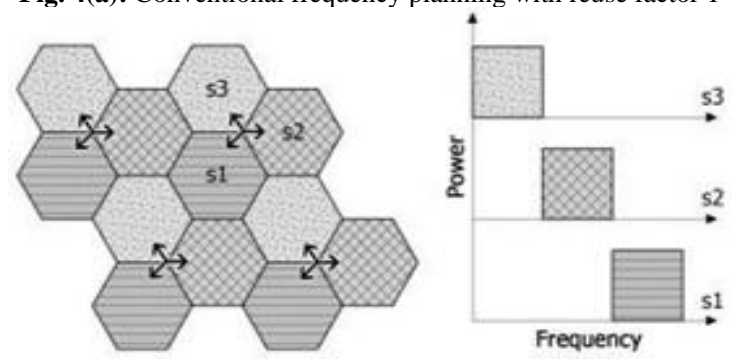

Fig. 4(b): Conventional frequency planning with reuse factor 3

\section{Fractional frequency reuse (FFR)}

Fractional frequency reuse scheme was proposed to overcome the shortcomings of conventional frequency reuse schemes. In FFRbased schemes, the users with a stronger signal quality use a lower reuse factor scheme (such as RF1) and the users having lower SINR use a higher reuse factor schemes (such as RF3).

\section{Partial frequency reuse (PFR)}

It isn't transfer speed productive to reuse a similar recurrence reuse factor (FRF) in the whole cell [9]. An answer for increment the SINR of cell edge clients, while great ghastly proficiency is being kept up, is to utilize a reuse factor more noteworthy than one for cell edge areas and RF1 for cell focus districts [10].
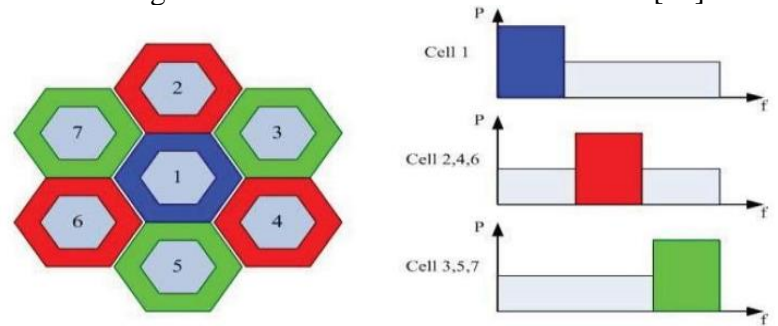

Fig. 5: Frequency planning and power allocation for PFR scheme

The essential plan of PFR is to place limitations on a locality of the assets therefore as a couple of assets don't seem to be utilized by some shopper categories by any means that. In PFR conspire as appeared in Fig.5, the combination accessible knowledge transfer capability is separated into four sub-groups. Cell focus UEs ar distributed within the repetition band utilizing reprocess issue of one. Cell edge UEs ar distributed within the reciprocal repetition band utilizing reprocess issue of three. This arrange is otherwise referred to as FFR-FI (FFR with full separation) because the phone edge shoppers ar altogether disconnected. As PFR doesn't utilize the complete accessible transfer speed, it prompts bring down cell output contrasted and RF1 plot.

\section{Soft Frequency reprocess (SFR)}

Delicate repetition reprocess conspire was projected in [8], it planned to keep up a strategic distance from the high between cell obstruction connected with reprocess issue one arrangement whereas larger ability is being given to the PFR plot. In SFR as appeared in Fig.6, each cell utilizes the combination accessible transmission capability. for each division, cell edge shoppers ar assigned within the portion of transfer speed with most noteworthy power level and cell focus shoppers ar selected with bring down power in no matter is left of the repetition band. RF1 is employed as a neighborhood|a district|a region|a locality|a vicinity|a section $\}$ of the cell focus area and FRF additional outstanding than one is employed at the cell edge locales.
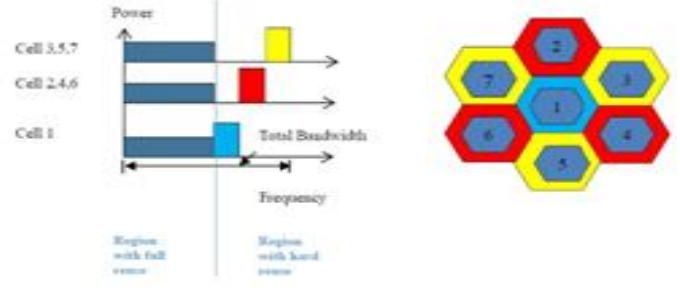

Fig. 6: Frequency planning and power allocation for SFR scheme

An improvement of SFR conspire is known as SFFR (Soft Fractional Frequency Reuse). The SFR and PFR strategies can improve the throughput of the clients at the cell edge locale by limiting the between cell impedance experienced by cell-edge clients. Notwithstanding, these plans may bring about lower cell throughput contrasted and ordinary RF1 plot. 


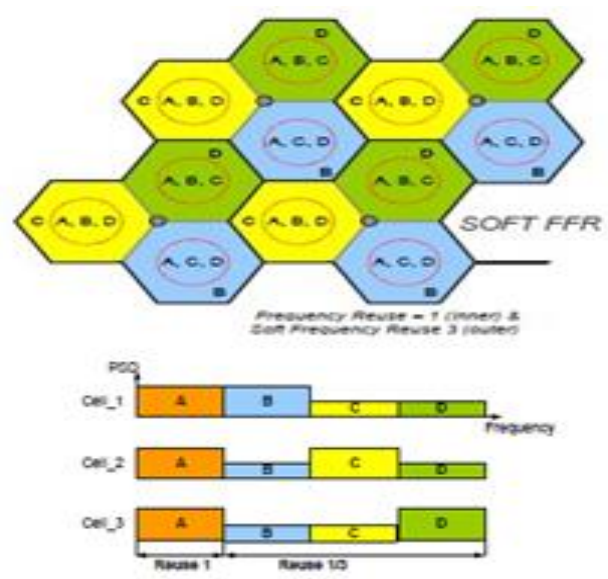

Fig.7: Frequency planning and power allocation for SFFR scheme

As PFR has bring down cell output contrasted and RF1 plot, in light-weight of the very fact that PFR doesn't utilize the whole information transfer capability accessible within the phone. Moreover, but SFR might enhance general framework limit as contrasted and PFR by use the whole accessible information transfer capacity; but SFR might prompt lower general framework limit than RF1 conspire. SFFR plot was planned to reinforce the overall cell output. In delicate FFR, the assets appointed to the purchasers at the cell edges of different cell square measure permissible to be used by alternate cells purchasers (inward) with some power limitation as appeared in Fig. 7.

\section{Intelligent reuse scheme}

In cagey utilise conspire, repeat band appointed to numerous areas grows relying upon existing workloads. At low employment, this set up begins with a RF3 like setup and then utilise issue are often adjusted with the augmentation of workloads for aiming to be PFR, SFR or RF1.

\section{Incremental frequency reuse}

IFR plot was planned to overcome many constraints of normal SFR set up, as an example, low vary productivity (because of the manner that the cell edge purchasers will utilize most extreme of $1 / 3$ of the whole information transmission), distended co-channel obstruction even at low activity stack circumstance, general cell limit misfortune once the framework is on top of half-full stacked. IFR intends to viably limit the ICI beneath low movement, whereas general framework limit is being sorted at an equivalent time. the first distinction amongst IFR and RF1 is and shortly thenceforth of the accessible information transmission IFR conspire begins quality allotment to the UEs. In IFR conspire, quality allotment of neighboring cells begins from numerous subchannels. As appeared in Fig.8, quality allotment of type A cells begins up from initial sub-channel, whereas type-B cells from $1 / 3$ of the combination accessible information transfer capability, and kind $\mathrm{C}$ cell from $2 / 3$ of the whole transmission capability. Albeit the bigger a part of the weaknesses of SFR set up are often inundated by IFR conspire, it simply offers higher execution beneath low activity circumstance.
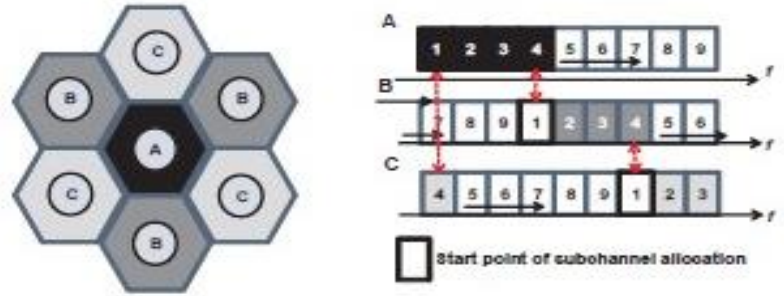

Fig. 8: Incremental frequency reuse (IFR) scheme

\section{Enhanced fractional frequency reuse (EFFR)}

EEFR was planned to in addition enhance the IFR and SFR framework execution. EFFR plans to create the framework limit particularly in over-burden movement circumstance. As appeared in Fig. 9, as IFR conspire, EFFR plot determines 3 cell-types for specifically bordering cells, and for each phone write a touch of the mixture band to be specific Primary section area unit saved. the first Segments need to be orthogonal. the remainder subchannels separated from the first section form Secondary section. within the meanwhile, the first section of a cell-type could be a piece of the Secondary Segments of different a pair of cell-types. All sub-directs in each cell's Primary section may be concerned by this cell voluntarily, but simply a touch of sub-diverts within the Secondary section may be possessed by this cell in between cell electric resistance aware means. each cell's Primary section is in addition isolated into a RF3 (reuse-3) section and RF1 (reuse-1) section. The RF1 half may be reused by all cell-types, tho' RF3 half may be reused by different same cell-type. The RF3 subchannels area unit embezzled to be reused by specifically connecting cells, that so lessens the co-channel impedances [11].

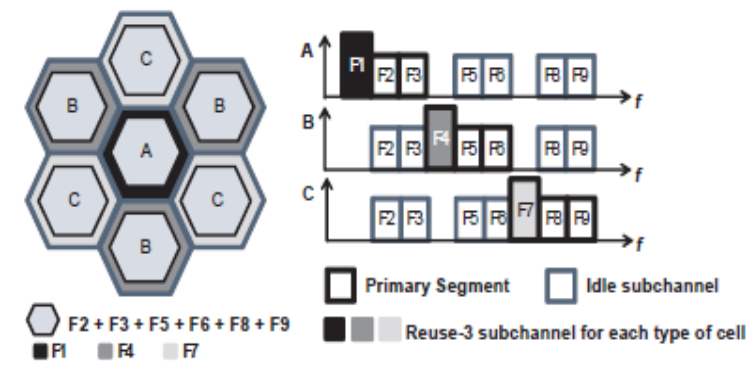

Fig. 9: Enhanced fractional frequency reuse (EFFR) scheme

\section{Semi-static schemes}

Some examples of semi-static ICI avoidance schemes are included in the following subsections.

\section{Siemen's proposal}

As appeared if Fig ten, in repetition recycle arrange of Siemen's proposition $[4,10]$, the mixture accessible transmission capability is partitioned off into $\mathrm{N}$ sub-groups. At that time $\mathrm{X}$ sub-groups square measure employed by the purchasers at the cell-edges, for instance, $\mathrm{X} \subseteq \mathrm{N}$. Also, the $\mathrm{N}-3 \mathrm{X}$ sub-groups square measure employed by the cell-focus purchasers. The $\mathrm{X}$ sub-groups used for the purchasers at the cell-edges of near cells square measure orthogonal to every alternative, whereas the $\mathrm{N}-3 \mathrm{X}$ sub-groups used for the purchasers at the cell-focuses square measure indistinguishable altogether cells. The sub-band use of purchasers at the cell-edges is received seeable of the activity stack. within the event that an additional sub-band is employed for the purchasers at the cell-edge, the sub-band used for cell-focus UEs are going to be fall by three sub-groups.
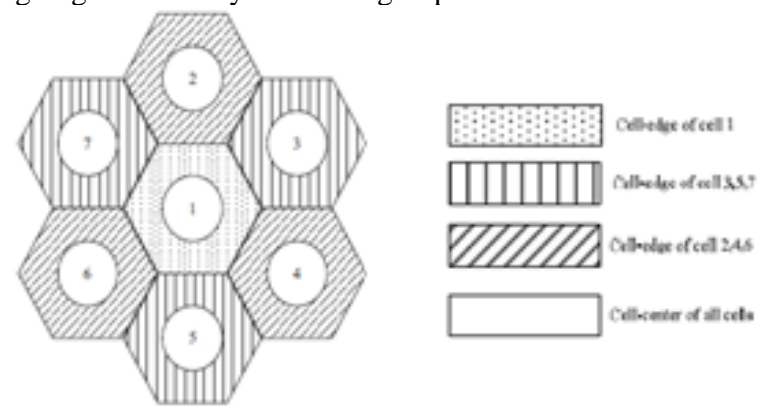

Fig.10: Frequency reuse scheme of siemen's proposal

Frequency reuse scheme, $X$ 
This set up planned in [12] depends on Ericsson's proposition of static return recycle set up and Siemen's proposition of semi-static ICIC. during this set up, a bit of the sub-groups is employed by cell-edge UEs with full power and therefore the entire vary is used by cell-focus shoppers with lessened power level of the subgroups that area unit accessible at the cell edge. On the off likelihood that the cell-edge territory of a selected cell seems to be smartly stacked, the cell will acquire the sub-groups sent in celledge zone of adjacent cells. The set up considers the variability of movement stack between cell-focus UEs and cell-edge UE and additionally the activity stack changes among adjacent cells.An case of X conspire is appeared in Fig. 11. The cell-edge of cell one has overwhelming activity stack whereas cell-edge movement stack is traditional in cell two, four and half dozen and low in cell three, 5 and 7. during this manner, the return sub-groups are going to be noninheritable from cell edge territory of cell three, five and seven by cell one. it's noticed that, the return quality should be obtained once the activity stack altogether the cell-edge zone of cell three, five and seven is low therefore the cell-edge UEs in neighboring cells cannot step in one another.

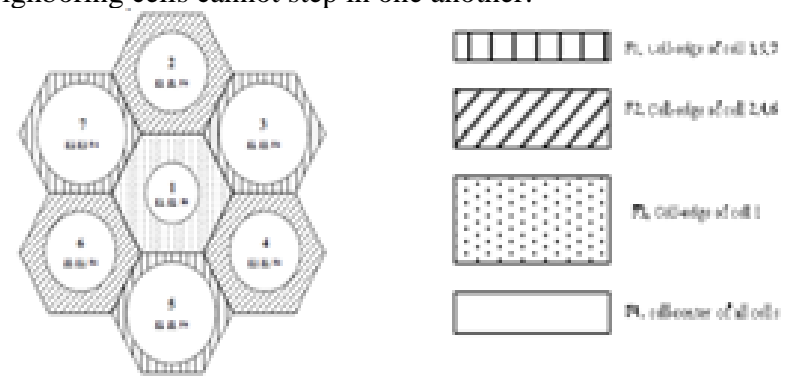

Fig. 11: Frequency reuse scheme of the proposal in [10]

\section{Dynamic schemes}

The scale and many-sided quality of gift day cell framework have forced the requirement to analyze the cell composed based mostly plans as potential models for overseeing such deeply complicated framework. The targets of the projected expedited plots in the main center to amplify general framework output, limit obstruction and to boot limit management use [6]. Coordination based mostly arrange are often delegated brought along, planned circulated, semi-appropriated and independent distributed arrange.

\section{Centralized theme}

In this arrange, coordination is well-kept by a unified controller that gathers all the channel state knowledge (CSI) of each shopper existing within the framework and doles out the accessible plus squares to every eNB specified limit is expanded . afterward, every eNB ought to forward the channel state knowledge got from each UE to the focal management unit and find back the plus portion knowledge. Be that because it might, it's laborious assignment to try and do unified designing on account of the rigorous time expected to trade booking knowledge and therefore the intensive input file needed by the shoppers to transmit all the Canadian Security Intelligence Service. that's the rationale, LTE-A framework has canceled the focal management unit and relied on coordination among eNBs over the X2 interface with no brought along coordination in an exceedingly level engineering [4].

\section{Semi-Distributed scheme}

In semi-dispersed plans, coordination is mostly existent at 2 levels, as an instance, the focal dominant substance level and therefore the eNBs level. Like brought along arrange, a focal dominant component is distributed that controls numerous eNBs. Be that because it might, in semi-conveyed approach, each supercasing of mass assets area unit selected to every eNB by the focal component although within the unified arrange the focal component distributes the channels specifically to each shopper on define premise. Consequently, in semi-dispersed arrange, every eNB is up to speed to dispense channels on the sting level to the shoppers that area unit served. because the plus designation assignment is distributed amongst eNBs and focal dominant substance, tired all the process heap of the arrange are often diminished. some cases of semi-conveyed plans are often found in [13-17]. The semi-disseminated arrange are often utilised for eICIC (improved put down Cell Interference Coordination) in HetNets (Heterogeneous Network) [6].

\section{Coordinated-Distributed scheme}

In unified and semi-appropriated approaches, all the electric resistance knowledge on every plus sq. ought to be gathered at the focal management substance, and truly, the quantity of this knowledge needed from eNBs to the focal controller are often usuriously expansive [15]. Thus, the speed of commerce knowledge amongst eNB and focal substance should be restricted, that brings concerning debased general framework execution. In composed disseminated plans, focal substance does not need to play out the coordination and assets area unit distributed simply at the eNB level. Be that because it might, even currently coordination between eNBs is needed for commerce CSI reports to stay up worldwide ICIC. For viable usage, the planned circulated plot is better in light-weight of the actual fact that it's clear aces over the semi-conveyed approach, as an instance, limiting time and drooping overhead due to the orderly correspondence amongst eNBs and focal controller, decreasing the many-sided nature of system framework as focal controller is done in here. some cases of expedited disseminated plans careful within the writing are often found in [12]. Fig. twelve demonstrates a case of composed circulated plot.

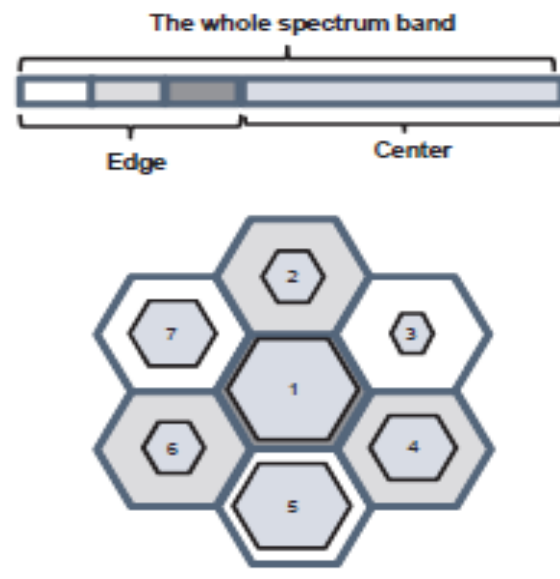

Fig. 12: Dynamic fractional frequency reuse scheme

A case of expedited sent plot wherever cell focus limits area unit modified more and more in sight of cell stack, shopper practices, and electrical phenomenon circumstance from border cells. As found in figure, the cell focus regions area unit numerous|of varied|of assorted \} size in various cell and cell one has high load whereas cell three has most borderline load. Thus, the district of RF1 comes larger in cell one as contrasted and cell three.

\section{Autonomous-Distributed scheme}

In autonomous-distributed approaches, resource allocation is made only at the eNB level without any use of focal dominant component for coordination. this can be the alikeness of selfruling disseminated plans with expedited distributed plans. Then again, not in the least like composed distributed plans, coordination between eNBs is not needed for self-sustaining disseminated plans. for every eNB, channels ar assigned by the $\mathrm{eNB}$ to its relating UEs relying upon the near information 
accumulated from its UEs. In self-ruling sent conspire, it's conceivable to place the RBs anywhere (in a distributed form) PRN to enhance the framework limit, that the framework goes concerning as self-arranging framework. therefore on continue organize wide ICIC and nice reasonableness with self-ruling disseminated plans, some of the RBs of every eNB should be restricted by limiting force level or to not utilize it in the least that so decreases the ICI on those RBs for near cells. Since, there's no coordination among eNBs in freelance plans, the RBs ought to be confined ar chosen supported SINR estimations of these RBs. Low SINR level indicates that Associate in Nursing plus sq. is being employed by contiguous cells. At the purpose once RBs ar being restricted by $\mathrm{eNB}$, an idea needs creating a trade off between the good thing about decreasing the ICI in neighboring cells and also the price of victimization the vary accessible [7]. Some case of freelance circulated ICIC plans may be found in [612].

\section{Interference randomization}

One of 3 ICI relief procedures is called as obstruction organisation. In obstruction organisation strategy, the clients' data ar unfold up finished Associate in Nursing confiscate set of subcarriers therefore ohmic resistance state of affairs may be randomised and repetition good selection acquire may be accomplished. In ohmic resistance organisation, in each cell the clients' data ar consecutively parceled out over a amount repetition lumps. At the purpose once all the asked for transmission ar assigned, subcarriers amendment is created in impulsive manner with the goal that each UE's transmission is self-assertively unfold up finished the mixture time-recurrence network. Fig. twelve demonstrates the allotment of subcarriers during a given cell once the pseudorandom amendment. In every meddling cell, the pseudorandom stage is performed freely. The cell explicit scrambling causes the ohmic resistance unfold up aboard the transmission of a given consumer. because the secret writing is performed at the transmitter amid transmission, the whole piece stream may be effortlessly recuperated at the lower than fascinating finish. As ohmic resistance framework needs no tired overhead for coordination among cells and fewer unpredictable plus administration, it's cheap for pragmatic framework [19].
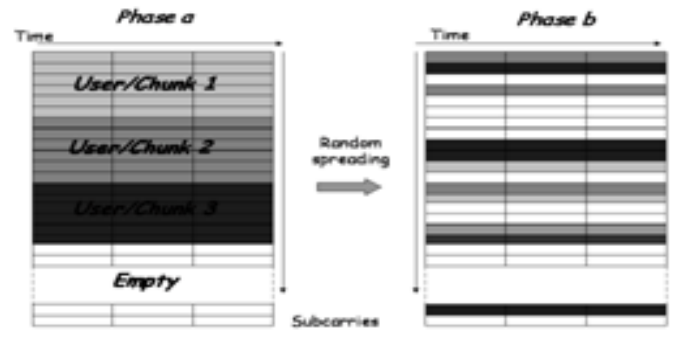

Fig. 13: Subcarrier allocation before (a) and after (b) random permutation

\section{Conclusion}

This paper introduces associate audit of 3 primary varieties of obstruction evasion plans, to be specific, static, semi-static and dynamic plans. Likewise the basic plan of ohmic resistance organisation is examined. Between cell obstruction coordination techniques is thought of as a booking arrange that considers the circumstance of near cells to diminish the result of between cell ohmic resistance (IC) and enhance the cell edge turnout. All in all, the ICI dodging plans place limitations of utilizing downlink assets, to Illustrate, time/recurrence/transmit management. The coordination of those confinements restrains the age of obstruction in an exceedingly cell organize. during this manner, at the lower than fascinating finish within the system scope, SINR is increased, that so offers an opportunity to obtaining swollen info rate over the system scope territory. During this paper, a review of ohmic resistance evasion plans utilised to alleviate the between cell obstruction issue happened in downlink LTE framework square measure thought of. Our future work incorporates to audit this ohmic resistance cancelation plans for LTE organize associated additionally to propose an obstruction moderation conspire for downlink LTE framework.

\section{Acknowledgement}

I am Very thankful to my guide Dr.N S Murti Sarma for his valuable suggestions to make this paper ready.

\section{References}

[1] Himayat N, Talwar S, Rao A \& Soni R. "Interference Management for 4G Cellular Standards", IEEE Communications Magazine, Vol. 48, No.8,(2010), pp.86-92.

[2] Daeinabi A, Sandrasegaran K \& Zhu X, "Survey of Intercell Interference Mitigation Techniques in LTE Downlink Networks", Australasian Telecommunication Networks and Applications Conference, (2012), pp.1-6.

[3] Necker MC, "A Novel Algorithm for Distributed Dynamic Interference Coordination in Cellular Networks", Proc. KiVS, (2011), pp.233-238.

[4] Bosisio R \& Spagnol U, "Interference Coordination vs. Interference Randomization in Multicell 3GPP LTE System", IEEE Wireless Communications and Networking Conference, (2008), pp.824-829.

[5] Boudreau G, Panicker J, Guo N, Chang R, Wang N \& Virzic S, "Interference Coordination and Cancellation for 4G Networks", IEEE Communications Magazine, Vol.47, No.4,(2009), pp.74-81.

[6] Hamza AS, Khalifa SS, Hamza HS \& Elsayed K, "A Survey on Inter-Cell Interference Coordination Techniques in OFDMA-Based Cellular Networks", IEEE Communications Surveys \& Tutorials, Vol.15, No.4,(2013), pp.1642-1670.

[7] Mao X, Maaref A \& Teo KH, "Adaptive soft frequency reuse for inter-cell interference coordination in SC-FDMA based 3GPP LTE uplinks", IEEE GLOBECOM Global Telecommunications Conference, (2008), pp.1-6.

[8] Ericsson, "Downlink inter-cell interference coordination/ avoidance evaluation of frequency reuse", Tech. Rep. TSG-RAN WG1 R1-061374, 3rd Generation Partnership Project (3GPP), (2006).

[9] Kwan R \& Leung C, "A Survey of Scheduling and Interference Mitigation in LTE", Electrical and Computer Engineering, (2010).

[10] Khan F, "LTE for 4G Mobile Broadband: Air Interface Technologies and Performance", Cambridge University Press, (2009).

[11] Zheng X \& Walke B, "Resource Allocation and Reuse for InterCell Interference Mitigation in OFDMA based Communication Networks", Wireless Internet Conference, (2010), pp.1-6.

[12] Fan X, Si C \& Zhang X, "An Inter-Cell Interference Coordination Technique Based on Users' Ratio and Multi-Level Frequency Allocations", International Conference on Wireless Communications, Networking and Mobile Computing, (2007), pp.788-802. 